\title{
Raman scattering and electrical conductivity in highly disordered activated carbon fibers
}

\author{
A. W. P. Fung \\ Department of Electrical Engineering and Computer Science, Massachusetts Institute of Technology, \\ Cambridge, Massachusetts 02139
}

A. M. Rao

Department of Physics, Massachusetts Institute of Technology, Cambridge, Massachusetts 02139

K. Kuriyama

Center for Materials Science and Engineering, Massachusetts Institute of Technology, Cambridge, Massachusetts 02139

M.S. Dresselhaus

Department of Electrical Engineering and Computer Science and Department of Physics, Massachusetts Institute of Technology, Cambridge, Massachusetts 02139

G. Dresselhaus

Francis Bitter National Magnet Laboratory, Massachusetts Institute of Technology, Cambridge, Massachusetts 02139

M. Endo

Department of Electrical Engineering, Faculty of Engineering, Shinshu University, Nagano 380, Japan

N. Shindo

Osaka Gas Co., Konohana-ku, Osaka 544, Japan

(Received 31 August 1992; accepted 28 October 1992)

Because of their unusually large specific surface area (SSA), Activated Carbon Fibers (ACF's) have a huge density of micropores and defects. The Raman scattering technique and low-temperature dc electrical conductivity measurements were used as characterization tools to study the disorder in ACF's with SSA ranging from $1000 \mathrm{~m}^{2} / \mathrm{g}$ to $3000 \mathrm{~m}^{2} / \mathrm{g}$. Two peaks were observed in every Raman spectrum for ACF's and they could be identified with the disorder-induced peak near $\sim 1360 \mathrm{~cm}^{-1}$ and the Breit-Wigner-Fano peak near $\sim 1610 \mathrm{~cm}^{-1}$ associated with the Raman-active $E_{2 g_{2}}$ mode of graphite. The graphitic nature of the ACF's is shown by the presence of a well-defined graphitic structure with $L_{a}$ values of $20-30 \AA$. We observed that the Raman scattering showed more sensitivity to the precursor materials than to the SSA of the ACF's. From $4 \mathrm{~K}$ to room temperature, the dc electrical resistivity in ACF's is observed to follow the $\exp \left[\left(T_{0} / T\right)^{1 / 2}\right]$ functional form and it can be accounted for by a charge-energy-limited tunneling conduction mechanism. Coulomb-gap conduction and $n$-dimensional $(n \leqslant 3)$ variable-range hopping conduction models were also considered but they were found to give unphysical values for their parameters.

\section{INTRODUCTION}

Activated Carbon Fibers (ACF's) are of great interest because they have a huge Specific Surface Area (SSA), exceeding that in other solids. A nominal SSA value up to $3000 \mathrm{~m}^{2} / \mathrm{g}$ is not beyond reach of the technology to date, as reported by at least one manufacturer of ACF's. Because of their unusually large SSA, ACF's are used in industry to build double-layer capacitors and as rechargeable battery-like devices. ${ }^{1}$ Like other active carbons, ACF's are also a good adsorption agent for solvents, vapors, and pollutants in gases as well as in water.
Besides their commercial interest, ACF's are also a good material for studying strong localization and disorder-related phenomena in the transport properties because of their unusually high density of defects. Although the adsorption properties of activated carbons have been extensively studied, ${ }^{2,3}$ the optical and the electrical properties of activated carbons have not been investigated with equal vigor. So far as optical spectroscopy is concerned, infrared experiments have been carried out for activated carbon, ${ }^{4,5}$ but only to the extent of determining the surface species and their physisorption and chemisorption characteristics. Our Raman study 
of this material, however, yields information about the intrinsic crystalline structure of the carbon system and helps to elucidate the electrical transport results.

There are few previous studies of the electrical conductivity of active carbon rods. Besides being limited to room-temperature measurements, these conductivity studies often focused on the adsorption characteristics of vapors and gases such as oxygen and water vapor, ${ }^{6,7}$ rather than on the intrinsic transport properties of this disordered carbon material. Since activated carbon had been successfully fabricated in fiber form, various transport properties of this disordered material were investigated, utilizing the greater measurement sensitivity provided by the fiber geometry. Di Vittorio ${ }^{8}$ made a preliminary survey of the temperature dependence of the dc electrical conductivity, the magnetoresistance, the thermal conductivity, and the thermopower of pitch-based ACF's with specific surface area (SSA) of $1000 \mathrm{~m}^{2} / \mathrm{g}$. Kuriyama and Dresselhaus ${ }^{9}$ have done a more thorough study of the de conductivity and the photoconductivity over a temperature range of $\sim 30 \mathrm{~K}<T<300 \mathrm{~K}$ for phenolic ACF's with SSA's ranging from $1000 \mathrm{~m}^{2} / \mathrm{g}$ to $2000 \mathrm{~m}^{2} / \mathrm{g}$. The conduction mechanism in disordered solids is generally known to be governed by hopping. To identify the hopping conduction mechanism, which is sensitively studied only at low temperatures, we measured the electrical conductivity down to $4 \mathrm{~K}$ in this work.

In what follows, we shall describe the fabrication and the structural properties of ACF's. We then demonstrate in the experimental section both a disorder-induced peak and a Raman-allowed peak in the Raman spectrum. We further show that the conductivity of ACF's follows the functional form $\sigma(T)=\sigma_{0} \exp \left[-\left(T_{o} / T\right)^{1 / 2}\right]$. Different hopping conduction models are evaluated in the discussion section below based on the numerical values for their physical parameters. We conclude that like granular metallic systems, to which the microstructure of ACF's can be compared, the conduction behavior of ACF's for the entire measurement temperature range $(4 \mathrm{~K}$ to $300 \mathrm{~K}$ ) can be best explained by the chargeenergy-limited tunneling conduction model.

\section{FABRICATION OF ACF'S}

The precursor materials of the ACF's we studied are isotropic pitch and phenol. The SSA's range from $1000 \mathrm{~m}^{2} / \mathrm{g}$ to $3000 \mathrm{~m}^{2} / \mathrm{g}$ for the pitch-based ACF's and from $1000 \mathrm{~m}^{2} / \mathrm{g}$ to $2000 \mathrm{~m}^{2} / \mathrm{g}$ for the phenolic ones. The precursor is first spun to form the fiber. Then, the fiber is prepared for activation in an antiflammable process at a temperature of 200 to $400{ }^{\circ} \mathrm{C}$. Finally, the fiber is activated. In the activation process, the fiber is heated in the temperature range $800-1200{ }^{\circ} \mathrm{C}$ for pitch-based fibers and $800-1100{ }^{\circ} \mathrm{C}$ for phenolic fibers in $\mathrm{O}_{2}, \mathrm{H}_{2} \mathrm{O}, \mathrm{CO}_{2}$, or other oxidizing atmospheres. The activation process for the phenolic ACF's used in this paper is described in Ref. 3. The main parameter that characterizes ACF's, the specific surface area (SSA), is controlled by the temperature and the time for activation, the activation process, and the precursor materials. The SSA is measured using BET analysis of the adsorption isotherms of $\mathrm{N}_{2}$ at $78 \mathrm{~K}$ and $\mathrm{CO}_{2}$ at $195 \mathrm{~K}^{3}$

The ACF's studied include three categories, namely pitch-based fibers (ACP), long phenolic fibers (FRL), and short phenolic fibers (FRS), where the manufacturer's designations are indicated in parentheses. Although the FRL fibers and the FRS fibers originated from the same precursor, they were treated differently by the manufacturer during their activation processes, resulting in differences in their microstructures so that the resultant degree of disorder in these two types of ACF's could be quite different. FRL fibers are typically more than $10 \mathrm{~cm}$ long and FRS fibers are generally shorter than $1 \mathrm{~cm}$. The ACP fibers, having a different precursor material, were expected to show yet a different microstructure.

\section{EXPERIMENTAL DETAILS}

The Raman scattering experiments were performed in the backscattered configuration. An argon-ion source was used to provide coherent radiation of less than $100 \mathrm{~mW}$ at a wavelength of $4880 \AA$. In order that the results be truly characteristic of the type of fibers under study, the laser beam was deliberately slightly defocused on the sample so that more fibers could be sampled and radiation heating could be avoided. The scattered beam from the sample was collected by a 50 $\mathrm{mm}$ camera lens into the entrance slit of a SPEX-1403 monochromator, the setting of which corresponded to a bandpass of $\sim 6 \mathrm{~cm}^{-1}$. This bandwidth is sufficiently narrow to preserve the line shape of the broad Raman peaks observed. To avoid fluorescence which would otherwise be produced if the samples were held in some transparent medium, such as a capillary tube or a sandwich of glass plates, we used parafilm strips to wrap the ends of the fiber bundle onto a glass plate substrate with the middle part of the fiber bundle conveniently exposed to the incident radiation. Samples made of short fibers about $1 \mathrm{~cm}$ long did not fall apart when prepared in this fashion because static electricity held the fibers together. A good signal-to-noise ratio was obtained after averaging each spectrum over six 25 -minute scans.

The transport experiment involved measurements of the resistance of single fibers over a range of temperature spanning $4.2 \mathrm{~K}$ to $300 \mathrm{~K}$ using the four-point probe method. Silver paint was used to establish contacts between the fiber resting on a glass plate and four copper wires placed in the proximity of the fiber. The contact 
resistance measured in this experiment was on the order of $10 \Omega$ typically and was much smaller than the sample resistance $(>1 \mathrm{k} \Omega)$.

The voltage across the middle contacts was measured by a Keithley model-181 digital nanovoltmeter while the current to the fiber was provided by a Keithley model-225 nanoampere source. Because the sample resistance could go up to tens of megaohms at low temperature, measurements of such high resistances required a few precautions. First, the current level was adjusted so that the power dissipated in the sample was less than $10^{-7} \mathrm{~W}$. A resistor of $\sim 10 \mathrm{M} \Omega$ was placed across the input terminals of the nanovoltmeter. At low temperature, it could divert the current from the sample so that the current source could now provide an output current above its minimum range without causing overflow in the nanovoltmeter. The resistor also reduced the total impedance seen by the nanovoltmeter so that a voltage correction was not necessary. Finally, neighboring samples were placed at least $5 \mathrm{~cm}$ apart, a distance observed to be long enough to avoid the problem of signal coupling of two large resistances at low temperature.

A temperature scan from $4.2 \mathrm{~K}$ to room temperature took place by natural warming. After the experiment, the resistivity of each sample was calculated using the diameter and length of the sample measured by Scanning Electron Microscopy (SEM).

\section{EXPERIMENTAL RESULTS}

Raman measurements were made on a series of FRL, FRS, and ACP fiber samples to examine the effect of the SSA, the precursor material, and its morphology on the Raman spectra. Shown in Fig. 1 are the Raman spectra for FRL20, FRS20, and ACP20 fibers and their

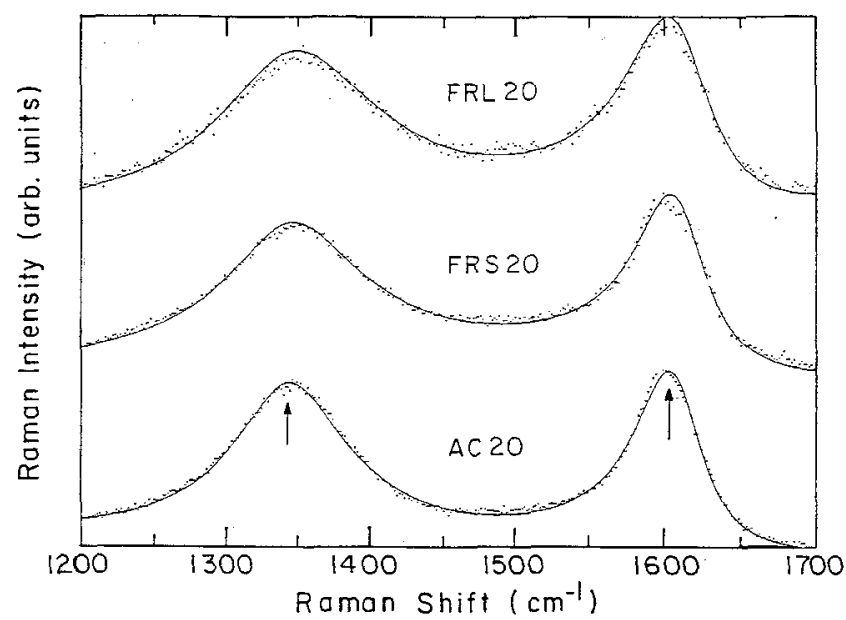

FIG. 1. Raman spectra for FRL20 (top figure), FRS20 (middle figure), and ACP20 (bottom figure) activated carbon fibers. The solid line is the fit to a BWF line at around $1610 \mathrm{~cm}^{-1}$ and a Lorentzian line at $\sim 1350 \mathrm{~cm}^{-1}$, indicated by vertical arrows. corresponding fitting curves. Each of these spectra is typical of all the fibers with different SSA's in the same fiber category. The alphabetic labels indicate whether the fibers are long phenolic, short phenolic, or pitch-based ACF's. The numbers in the labels represent the SSA divided by $100 \mathrm{~m}^{2} / \mathrm{g}$, so that $\mathrm{SSA}=2000 \mathrm{~m}^{2} / \mathrm{g}$ for all the ACF's in Fig. 1.

Two peaks were observed in the Raman spectrum for each of the 10 samples studied. The best fit to the data was obtained with a Lorentzian fit to the broad line near $1360 \mathrm{~cm}^{-1}$ and a Breit-Wigner-Fano (BWF) line shape around $1610 \mathrm{~cm}^{-1}$. The latter line shape results from the interaction of a Raman-active continuum with the discrete Raman-allowed mode, which can be identified as the $1582 \mathrm{~cm}^{-1} E_{2 g_{2}}$ mode in HOPG. The BWF line shape was also observed in the Raman spectra for other disordered graphitic systems, such as ionimplanted graphite ${ }^{10}$ and stage- 1 alkali metal graphite intercalation compounds $\mathrm{C}_{8} \mathrm{~K}, \mathrm{C}_{8} \mathrm{Rb}$, and $\mathrm{C}_{8} \mathrm{Cs}{ }^{11}$ The BWF line shape is described by the following expression:

$$
I(\omega)=\frac{I_{0}\left[1+\left(\omega-\omega_{0}\right) / q\right]^{2}}{1+\left[2\left(\omega-\omega_{0}\right) / \Gamma\right]^{2}}
$$

where $I(\omega)$ is the intensity as a function of frequency, $I_{0}$ the peak intensity, $1 / q$ the interaction between the discrete $E_{2 g_{2}}$ mode and the Raman-active continuum, $\omega_{0}$ the phonon peak frequency, and $\Gamma$ the full-widthat-half-maximum intensity (FWHM) of the unweighted Lorentzian (for which $q \rightarrow \infty$ ). The broad peak near $1360 \mathrm{~cm}^{-1}$ is disorder-induced, and has also been observed in other disordered graphitic systems such as benzene-derived carbon fibers. ${ }^{2}$ Analysis of these two lines yields values for the central frequencies $\left(\omega_{0}\right)$, the FWHM's $(\Gamma)$, and the relative integrated intensities $I_{1360} / I_{1580}$ of the disorder-induced peak near $1360 \mathrm{~cm}^{-1}$ to the BWF peak around $1610 \mathrm{~cm}^{-1}$, as well as values for $\Gamma_{1580} / q$ and the dimensionless interaction parameter $(1 / q)$ for the Raman-active mode with the BWF line shape. The Raman-active $E_{2 g_{2}}$ mode has a peak at $\sim 1580 \mathrm{~cm}^{-1}$ in single crystal graphite. The results are summarized in Table I.

We also measured the electrical conductivities for three long phenolic fibers (FRL10, FRL15, and FRL20) and three short ones (FRS12, FRS15, and FRS20). The temperature dependence of the conductivities of these phenolic ACF's is shown in Fig. 2, together with their best fits to the charge-energy-limited tunneling conduction model. The error bars for the data are about $20 \%$ of the absolute value and are mainly due to uncertainty involved in measuring the diameter of a nonuniformly thick fiber. A typical diameter for a phenolic ACF is about $10 \mu \mathrm{m}$. It is to be noted that while the values of the conductivities relative to the room-temperature value 
TABLE I. Listed are the fitting parameters obtained from fitting a Lorentzian line at $\sim 1350 \mathrm{~cm}^{-1}$ and a BWF line at $\sim 1610 \mathrm{~cm}^{-1}$ to the Raman data. The numbers in the fiber labels indicate their specific surface area in $100 \mathrm{~m}^{2} / \mathrm{g}$.

\begin{tabular}{lllllllllll}
\hline \hline Parameters & FRL10 & FRL15 & FRL20 & FRS12 & FRS15 & FRS20 & ACP10 & ACP15 & ACP20 & ACP30 \\
\hline$\left(\omega_{0}\right)_{1360}$ & 1348 & 1350 & 1350 & 1348 & 1348 & 1347 & 1347 & 1345 & 1345 & 1342 \\
$\Gamma_{1360}$ & 152 & 156 & 141 & 120 & 124 & 118 & 114 & 113 & 102 & 103 \\
$\left(\omega_{0}\right)_{1580}$ & 1611 & 1612 & 1611 & 1606 & 1612 & 1610 & 1610 & 1607 & 1609 & 1606 \\
$\Gamma_{1580}$ & 67 & 64 & 68 & 61 & 61 & 57 & 57 & 57 & 54 & 56 \\
$1 / q$ & -0.23 & -0.19 & -0.23 & -0.10 & -0.18 & -0.18 & -0.17 & -0.16 & -0.16 & -0.17 \\
$\Gamma_{1580} / q$ & -15 & -12 & -16 & -6 & -11 & -10 & -10 & -9 & -9 & -10 \\
$I_{1360} / I_{1580}$ & 1.89 & 1.90 & 1.92 & 1.67 & 1.69 & 1.70 & 1.69 & 1.81 & 1.70 & 1.84 \\
\hline \hline
\end{tabular}

are adequate to describe the temperature dependence, knowledge of the absolute values of the conductivities of various ACF's is important for making comparisons between these fibers.

It is suggestive to make a comparison between the room-temperature (RT) conductivity of ACF's and those of other carbon systems because systems with conductivities of about the same magnitude at RT may exhibit the same kind of conducting behavior at low temperature. A typical range of RT conductivity for phenolic ACF's is approximately $5-10 \mathrm{~S} / \mathrm{cm}$, which is comparable to that for active carbon rods, ${ }^{7}$ Saran carbon, ${ }^{13}$ and glassy carbon. ${ }^{14}$ ACF's are much more conductive than evaporated carbon thin films ${ }^{15}$ and a great deal less conductive than vapor-grown carbon fibers. ${ }^{16}$ At low temperature thin carbon films have been reported to conduct by 2-dimensional variable-range hopping conduction (VRHC) and at high

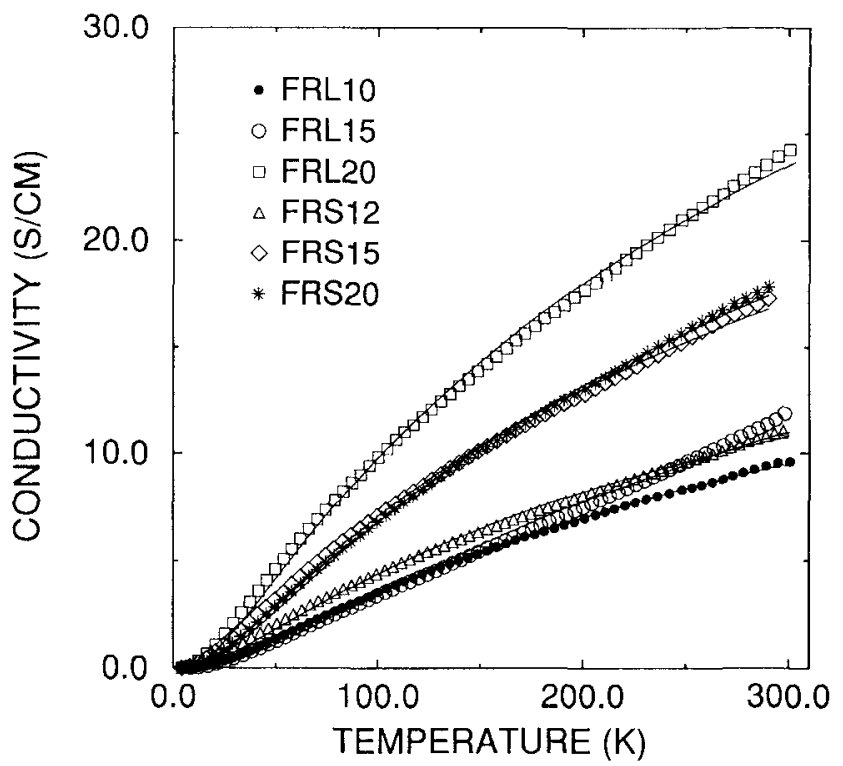

(a) temperature by the thermal activation of carriers. ${ }^{17}$ The temperature dependence of the conductivity of glassy carbon, however, has been reported to follow the behavior of 3-D VRHC, with some corrections at low temperature. ${ }^{18}$

VRHC is generally governed by Mott's law in the form of ${ }^{19}$ :

$$
\sigma(T)=\sigma_{0} \exp \left[-\left(\frac{T_{0}}{T}\right)^{1 / p}\right]
$$

where $\sigma_{0}$ is a characteristic conductivity constant, $T_{0}$ a fitting parameter sensitive to the energy needed for hopping, and $p=d+1$ with $d$ equal to the dimensionality of the system. The best fit to the measured $\sigma(T)$ is with $p=2$, as is also supported by the comparison of the plots of the logarithm of the electrical resistivity against $1 / T, 1 / T^{1 / 2}$ (Fig. 3), $1 / T^{1 / 3}$, and $1 / T^{1 / 4}$ (not shown)

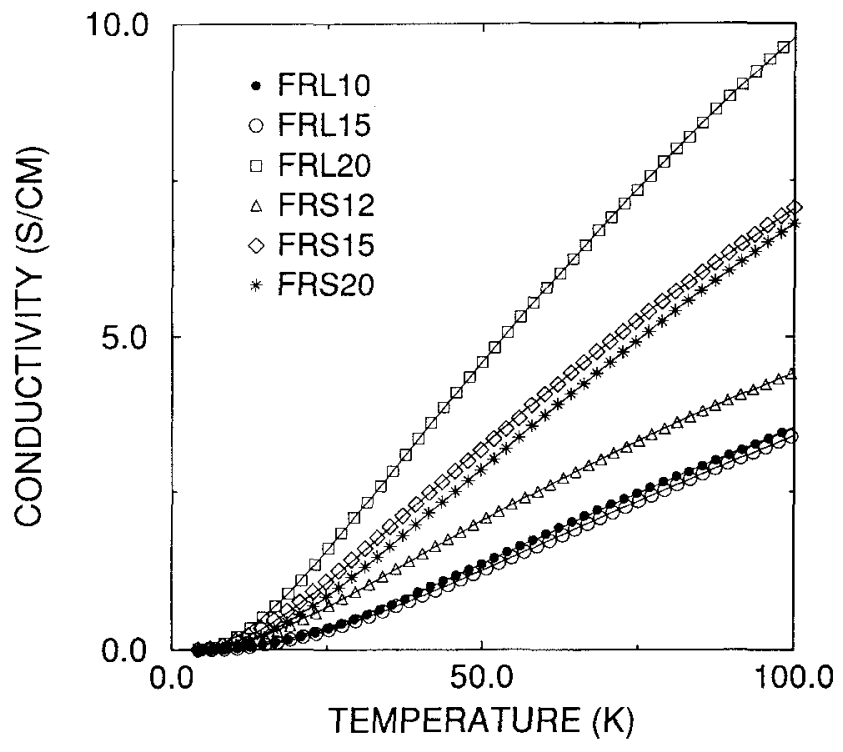

(b)

FIG. 2. Temperature dependence of the conductivity for phenolic ACF's with the listed specific surface area values and the fits using the charge-energy-limited tunneling model (a) over the entire measurement temperature range $4 \mathrm{~K}<T<300 \mathrm{~K}$ and (b) over $4 \mathrm{~K}<T<100 \mathrm{~K}$, where the $p$ values are very close to 2 [see Eq. (2)]. 


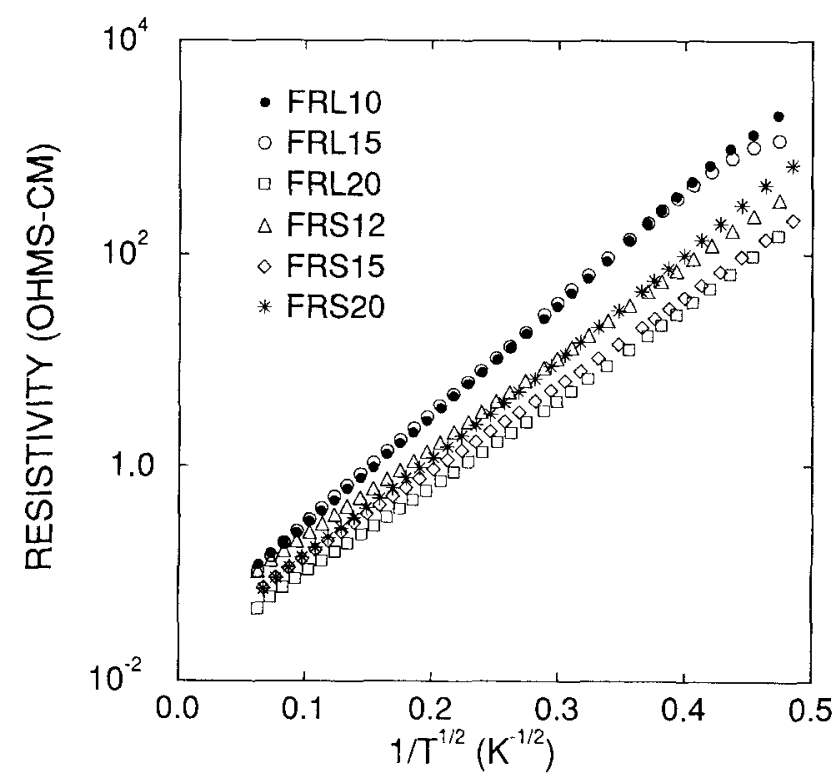

FIG. 3. The logarithm of the resistivity is plotted against $1 / T^{1 / 2}$ for the phenolic ACF's. A good linear fit is obtained over the temperature range $4 \mathrm{~K}<T<\sim 300 \mathrm{~K}$. The linearity of the curve over a wide temperature range might be evidence for the charge-energy-limited tunneling conduction model.

for all phenolic ACF's. We show only the $1 / T^{1 / 2}$ plots because they are the most linear curves over the entire measurement temperature range.

Because of the near linearity observed at the lowtemperature portion of almost all $1 / T^{1 / p}$ plots for ACF's and for other disordered materials previously reported in the literature, there is some ambiguity in the value for $p$ in Eq. (2). Following Hill ${ }^{20}$ and Zabrodskii, ${ }^{21}$ we can obtain the value for $p$ more quantitatively by plotting $\log T$ against the local activation energy $e_{a}$ defined by:

$$
e_{a} \equiv \frac{d(\log \rho)}{d\left(\mathrm{k}_{\mathrm{B}} T\right)^{-1}}
$$

where $\mathrm{k}_{\mathrm{B}}$ is the Boltzmann constant. De-emphasizing the large fluctuation in the high-temperature portion of the plot, induced by the process of differentiation, we obtained the values for $p$ in Table II using a robust linear fit. ${ }^{22}$ All the listed $p$ values center around 2 , consistent with the linearity in the $1 / T^{1 / 2}$ plot.

Di Vittorio et al. have examined ${ }^{8}$ the temperature dependence of the dc electrical conductivity of ACP10 fibers (where SSA $=1000 \mathrm{~m}^{2} / \mathrm{g}$ ) over the temperature range $25 \mathrm{~K}<T<300 \mathrm{~K}$ and found that it follows a
2-D VRHC model. Their conductivity data were extracted and analyzed according to our previous procedure. Comparison of the different $1 / T^{1 / p}$ plots yields $p=3$, in agreement with their conclusion, ${ }^{8}$ but the $1 / T^{1 / 2}$ plot has almost as good a linear fit. Furthermore, the $e_{a}$ plot in accordance with Eq. (3) yields $p=2$, which is also listed in Table II. In fact, it was found that nonlinearly fitting our $\sigma(T)$ data for phenol-based ACF's to Eq. (2) over a lower temperature range (e.g., $T<100 \mathrm{~K}$ ) always yields a value of $p$ closer to 2 . Hence, the $p=3$ value reported in Ref. 8 could be a result of their lack of data below $20 \mathrm{~K}$.

From a physical standpoint, ACF's are a complex material (their heterogeneity is indicated by the broad linewidths of the Raman-allowed line near $1600 \mathrm{~cm}^{-1}$ ) and there may be more than one conduction mechanism at work. In this connection, it is possible that the log plots for 2-D and 3-D VRHC might deviate from being linear because some other conduction mechanism may set in at low temperature (e.g., thermal activation from the Fermi level to the mobility edge if there is such an edge). We considered 2-D VRHC as a conduction mechanism in ACF's although strongly disordered systems tend to be isotropic because the layered structure may remain intact on a very small spatial scale while the distribution of these 2-dimensional structures is isotropic at long distances.

When we added an activation term $\sigma_{1} \exp \left(-\epsilon_{1} /\right.$ $\mathrm{k}_{\mathrm{B}} T$ ) in Eq. (2) in a nonlinear fit to the conductivity data, we found that although the contribution from the thermal activation term to $\sigma(T)$ in Eq. (2) is relatively unimportant ( $<7 \%$ for $p=2$ and $<22 \%$ for $p=3$ at $30 \mathrm{~K}$ ), it is definitely needed to achieve a good fit to our $\sigma(T)$ data. However, the fitting values for $\sigma_{1}$ are much smaller than required by Mott's minimum metallic conductivity given by ${ }^{19}$ :

$$
\sigma_{1}=0.03 e^{2} /\left(\hbar L_{i}\right)
$$

where $L_{i}$ is the inelastic scattering length, taken to be no larger than the average diameter of a graphite platelet (i.e., $<30 \AA$ ). Furthermore, the observed decrease in the activation energy $\epsilon_{1}$ with increasing disorder cannot be explained by this model. We therefore think that this activation term is not real and should not be included in our fits. Yet without this activation term, VRHC models cannot fit our $\sigma(T)$ data.

To ensure that the system is in strong localization, a constant de conductivity term $\sigma(T=0)$ was also

TABLE II. Values for the numerical constant $p$ in the exponent of Eq. (2) as determined by a robust fit to the log-log plot of the temperature dependence of the local activation energy. The conductivity data for the ACP10 fibers were extracted from Ref. 8.

\begin{tabular}{cccccccc}
\hline Fiber & FRL10 & FRL15 & FRL20 & FRS12 & FRS15 & FRS20 & ACP10 \\
\hline$p$ (robust fit) & 1.8 & 2.1 & 1.9 & 2.0 & 2.0 & 1.9 & 2.2 \\
\hline
\end{tabular}


included in the fit to Eq. (2) but the fitting values for $\sigma(T=0)$ were always within the fitting uncertainty. ${ }^{23}$

The best fits of the $\sigma(T)$ data for all phenolic ACF's to Eq. (2) over the range $4 \mathrm{~K}<T<300 \mathrm{~K}$ were obtained with $p=2$ and are shown in Fig. 2(a). Because the least-square value for $p$ becomes closer to 2 when the fit is over temperatures below $100 \mathrm{~K}$, we also fit our data only over this temperature range to obtain more accurate values for the fitting parameters, which were generally found to change by less than $30 \%$ from the original values. The fit for $T<100 \mathrm{~K}$ is shown in Fig. 2(b) and the corresponding fitting parameters are listed in Table III. The $1 / T^{1 / 2}$ scale in Fig. 3 favors a linear fit for the low-temperature region, the results of which give values consistent within $10 \%$ with those listed in Table III.

With $p=2$ in Eq. (2), there are actually two other possible conduction mechanisms besides 1-D VRHC, namely the Coulomb-gap conduction and the chargeenergy-limited tunneling conduction. The relevance of each of these models to the ACF system will be discussed in more detail in the next section.

\section{DISCUSSION}

From Table I, all the fitting parameters for the Raman experiment are extracted and plotted in Figs. 4-7 as a function of the SSA for the three classes of fibers. As will be discussed below, all the fitting parameters, when plotted against SSA, show little sensitivity to a change in SSA, but they are all sensitive to the types of ACF's. The solid lines drawn to connect the points in each plot versus SSA are intended to provide a guide to the eye and not a fit to the data.

The FWHM of the disorder-induced line $\left(\Gamma_{1360}\right)$ and that of the Raman-active line $\left(\Gamma_{1580}\right)$ are plotted, respectively, in Fig. 4 and Fig. 5 versus the SSA for the various kinds of fibers. The error bars in both figures are typically taken to be $10 \%$ of the linewidths. In view of the large linewidths of the two lines in each spectrum, we conclude that $\Gamma_{1360}$ and $\Gamma_{1580}$ are both almost independent of SSA. The change with different types of ACF's is, however, larger than the error bar in both figures. The linewidths for the FRL fibers are larger than the ones for the FRS fibers, which in turn are larger than those for the ACP fibers, indicating that the FRL fibers are more inhomogeneously disordered than

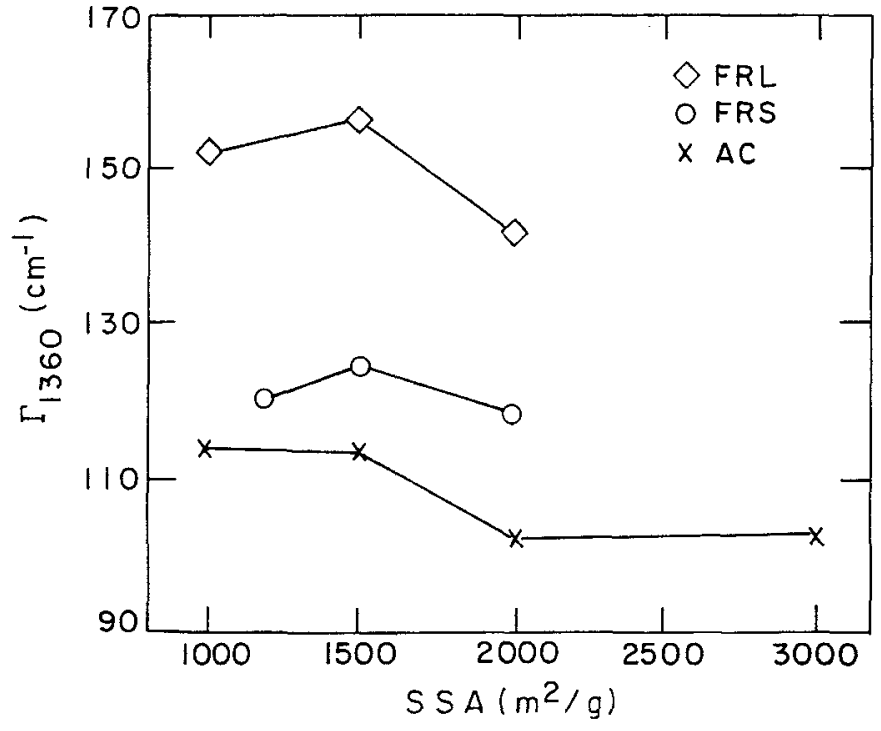

FIG. 4. The full-width-at-half-maximum intensities (FWHM) of the disorder-induced line at $1350 \mathrm{~cm}^{-1}$ for each type of activated carbon fiber are plotted as a function of the specific surface area.

the FRS fibers and that the ACP fibers have the smallest degree of disorder of all.

The Fano interaction coefficient $(1 / q)$, which is a measure of the asymmetry of the Raman-active peak and thus also of the degree of disorder, is plotted against SSA in Fig. 6. No simple trend is observed in the plot against SSA, but the trend of disorder versus fiber type in Fig. 6 generally agrees with Figs. 4 and 5 .

The ratio of the integrated intensity of the disorderinduced line at $1360 \mathrm{~cm}^{-1}$ to that of the Raman-active line at $1580 \mathrm{~cm}^{-1}(R)$ is plotted as a function of SSA in Fig. 7. The microcrystallite size of a graphitic system can be determined from the Raman spectra using the empirical formula given by ${ }^{24}$ :

$$
L_{a}(\AA)=\frac{44}{R} .
$$

It is interesting to see that the ratios $R$ for ACF's are always larger than 1.65 , whereas those for all the disordered carbon-based materials listed in Ref. 24 for finding Eq. (5) are below 1.5. The crystallite size is thus estimated to be $\sim 23 \AA-26 \AA$ from extrapolation. In this limit, the Raman scattering characterization technique becomes less sensitive, and this may explain the insensitivity of the linewidths and the Fano $1 / q$ parameter to

TABLE III. Values for the parameters obtained from the fit to the conductivity data using Mott's law with $p=2$.

\begin{tabular}{lcccrrr}
\hline \hline Fiber & FRL10 & FRL15 & FRL20 & FRS12 & FRS15 & FRS20 \\
\hline$\sigma_{0}(\mathrm{~S} / \mathrm{cm})$ & 35 & 37 & 61 & 29 & 47 & 55 \\
$T_{0}(\mathrm{~K})$ & 530 & 580 & 330 & 350 & 360 & 430 \\
\hline \hline
\end{tabular}




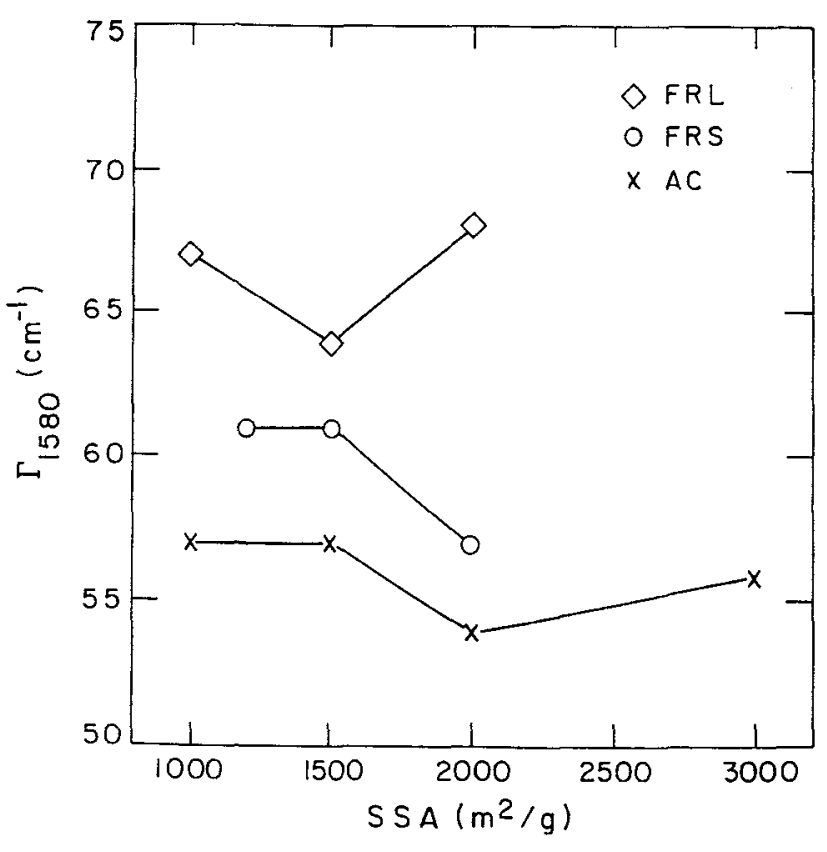

FIG. 5. The full-width-at-half-maximum intensities (FWHM) of the Raman-allowed peak at $1610 \mathrm{~cm}^{-1}$ for each type of activated carbon fiber are plotted as a function of the specific surface area.

SSA in all the previous plots. The positive slopes of the straight lines resulting from a linear fit in the same figure suggest that disorder increases with SSA and that unlike other characterization parameters, $R$ is sensitive to SSA. Although the plots in Fig. 7 still show that the FRL fibers have the largest degree of disorder among the activated carbon fibers reported here, the curve for the ACP fibers

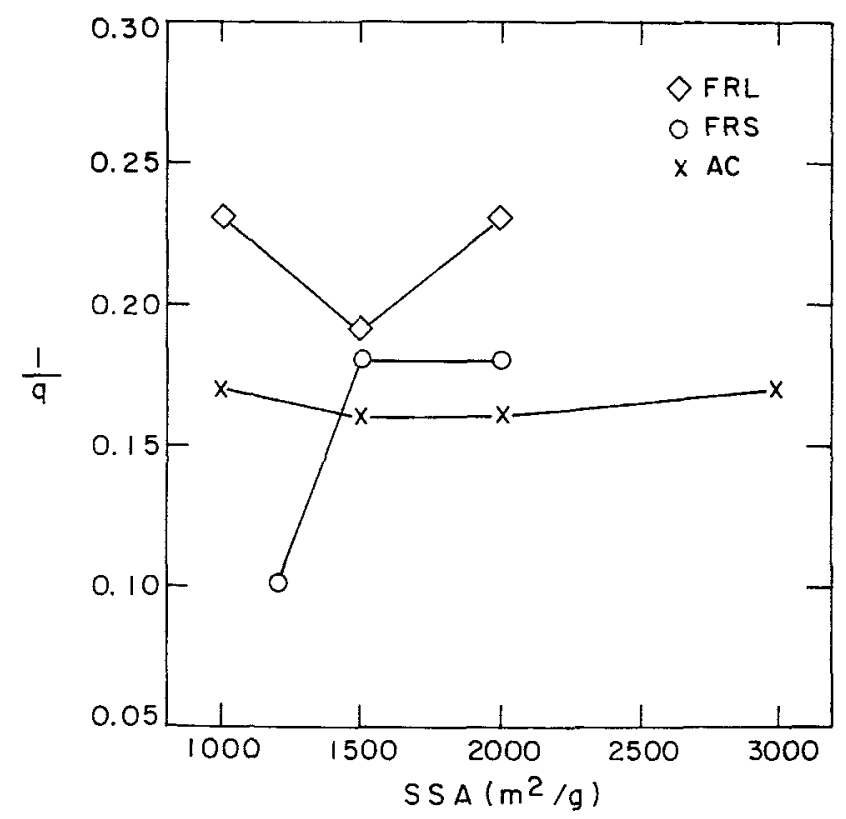

FIG. 6. The Fano interaction parameters $1 / q$ for the Raman-active mode for each type of fiber are plotted against the specific surface area.

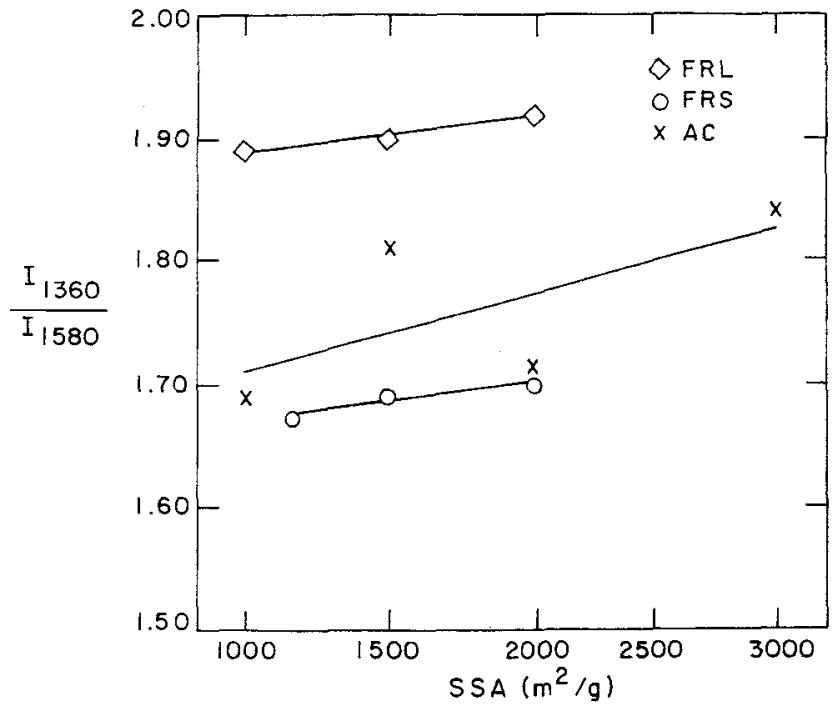

FIG. 7. The ratios of the integrated intensity of the disorder-induced peak to that of the Raman-active mode $\left(I_{1360} / I_{1580}\right)$ for each type of fiber are plotted as a function of the specific surface area.

is now above the FRS curve, unlike the previous plots discussed earlier. For large amounts of disorder, the relations between the linewidths and disorder may break down because many mechanisms can now contribute to the linewidths. On the other hand, $R$ may be more directly related to the degree of disorder because the $1360-\mathrm{cm}^{-1}$ peak is completely disorder-induced and so $R$ may be a more reliable characterization parameter for the degree of disorder than the linewidths.

Although not shown, the center frequency of the Raman mode is precursor-sensitive but SSA-insensitive. In all the ACF's studied here, the Raman mode appears above $1605 \mathrm{~cm}^{-1}$, indicating strong disorder in these fibers. ${ }^{25}$ The upshift from the HOPG value $\left(1582 \mathrm{~cm}^{-1}\right)$ could be due to the following reasons.

1. Contributions from the graphitic peak near $1582 \mathrm{~cm}^{-1}$ may overlap with those from the disorderinduced peak near $1620 \mathrm{~cm}^{-1}$.

2. Both the charge transfer effect and the chargetransfer-induced lattice contraction effect, as similarly observed in acceptor-doped Graphite Intercalation Compounds, ${ }^{11,26}$ could still play some role in producing a frequency shift of the graphitic peak in ACF's since ACF's are observed to be $p$-type at room temperature. ${ }^{9}$ The calculations for the frequency shift in Ref. 26, however, cannot be taken literally for ACF's that have localized states instead of conduction states. The microcrystallites may even be strained originally because of some other effects such as the strain associated with interactions between small microcrystallites.

Before we discuss our conductivity results, $x-$ ray $^{9}$ and TEM results are reviewed, along with the proposition of two simple models that can explain the high 
SSA observed in ACF's, so that a picture of the microstructure of ACF's can be drawn and then used to analyze the transport results. First, the very presence of a well-resolved graphitic peak in the Raman spectra indicates that the microcrystallites are quite graphitic in structure. We also believe the multiple layers in a single graphene platelet in the $1000 \mathrm{~m}^{2} / \mathrm{g}$ ACF's are almost certainly turbostratic in structure, on the basis of $\mathrm{x}$-ray diffraction results. ${ }^{9}$ The dissociation of the neighboring layers allows us to propose our first simple model to explain the high SSA observed in ACF's. In this model, ACF's are described as sheets of graphene platelets, and the thickness $(t)$ of one such graphite platelet is:

$$
t=\frac{2}{(\mathrm{SSA}) \rho}
$$

where $\rho=2.25 \mathrm{~g} / \mathrm{cm}^{3}$ is the density of graphite. For SSA increasing from $1000 \mathrm{~m}^{2} / \mathrm{g}$ to $3000 \mathrm{~m}^{2} / \mathrm{g}$, the mean thickness of each platelet then goes approximately from three graphite layers to one graphite layer when we take the thickness of one graphite layer to be $3.35 \AA$.

According to this simple model, a graphite platelet inside a $3000 \mathrm{~m}^{2} / \mathrm{g}$ fiber can only be one layer thick. However, we should also take note that first, the SSA obtained from BET measurements could be an overestimate because of multiple-layer gas adsorption. In addition, as a platelet gets smaller, the contribution to adsorption from the increasing proportion of the perimeter (edge) carbon atoms in the platelet becomes important. Secondly, to hold a fiber together, there need to be some thicker platelets. The $\mathrm{x}$-ray diffraction pattern ${ }^{9}$ shows extraordinarily broad linewidths, probably resulting from the contributions from the various platelets of different thicknesses. The average platelet thickness of about $10 \AA$ determined by the simple Scherrer analysis ${ }^{25}$ should also be considered only as an order-of-magnitude estimate. ${ }^{9}$

The Transmission Electron Microscopy (TEM) picture shown in Fig. 8, though for a pitch-based ACF, is believed to be typical of the microstructure of ACF's. In this picture, the length of an elongated platelet in the longitudinal direction $(a)$, which is believed to be the in-plane crystallite size $L_{a}$, is about $30 \AA$ while the length in the transverse direction $(b)$ is about $10 \AA$. While $b$ could be the thickness of a platelet, it might also be the transverse length of a noncircular platelet or the projection of a platelet oriented with its c-axis not normal to the incident electron beam. Consequently, a mere look at a TEM picture without quantitative image analysis can only give an order-of-magnitude estimate of the thickness of a platelet, but it confirms the existence of graphite platelets in ACF's and gives a value for $L_{a}$ consistent with the Raman results.

Our simple model is not capable of explaining the maximum SSA $\left(5000 \mathrm{~m}^{2} / \mathrm{g}\right)$ reported for ACF's in powder or granular form obtained from some kind of

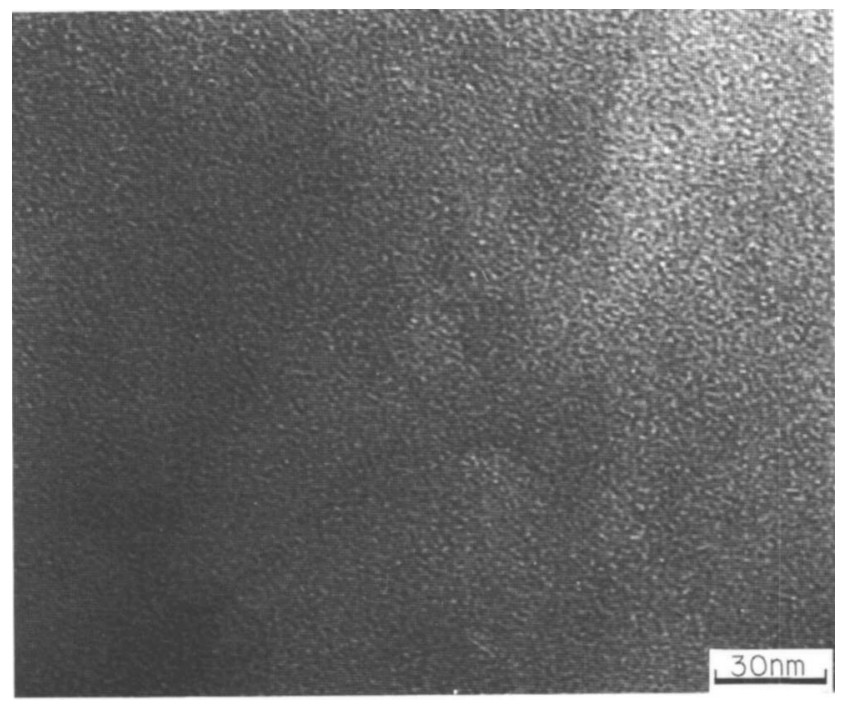

FIG. 8. The TEM picture shows that the size of a micropore in ACP30 (a pitch-based ACF with $\mathrm{SSA}=3000 \mathrm{~m}^{2} / \mathrm{g}$ ) is $\sim 8-30 \AA$. A graphite platelet, as estimated from Raman scattering, is about the same size.

pitch precursors. ${ }^{27}$ It is worth noting that this SSA value is not measured by the $\mathrm{N}_{2}$ adsorption method, as the entry to a micropore eventually becomes too small to admit an $\mathrm{N}_{2}$ molecule, but rather is measured by the capacitance method.

We will now discuss another highly simplistic model that can explain SSA's in excess of $3000 \mathrm{~m}^{2} / \mathrm{g}$. For a graphite platelet to have an SSA above $3000 \mathrm{~m}^{2} / \mathrm{g}$, it must have pores within itself. An extension from the previous model based on Eq. (6) is possible if we consider how the platelets are formed in the first place. We propose that the structure before activation could be a web of graphene ribbons intertwined in some random fashion. As an oxidizing reagent is introduced into the system, it strips the carbon atoms from the graphite web along its path, thus creating a worm-like tunnel of pores in the graphite web. Whenever there are a sufficiently large number of pore tunnels that percolate in the web, fragments of the graphite web will then break off from the main web. This process will continue to break down the main web and its fragments even further, resulting in a remnant that consists of a large number of small graphite platelets. Inevitably, these graphite fragments should now contain very small pores in their microstructures.

The smallest theoretical pore within a platelet is formed by a single atomic vacancy. Such a one-atom vacancy is now modeled as the space bounded by the perpendicularly bisecting surfaces between nearestneighboring atomic distances in all directions, including the c-axis direction. This elemental vacancy is then a pore that is covered by two triangular surfaces at the top and bottom (each with area $S_{t}$ ), and three rectangular 
side surfaces (each with area $S_{s}$ ). Both 1-layer and 2layer graphite platelets are supposed to be present. The pores on the two graphene layers are very likely to overlap and have the same size according to the wormweb model. Therefore, for a platelet $n$ layers thick with a number $N$ of $M$-atom clusters stripped from each layer, the SSA becomes:

$$
\mathrm{SSA}=\frac{2 S-2 N M S_{t}+n N(M+2) S_{s}}{\rho_{m} n c S-n N M m}
$$

where $S$ is the surface area of one $c$-surface of the platelet, $\rho_{m}$ the mass density of graphite, $c$ the interlayer distance in pure graphite, and $m$ the mass of a carbon atom.

In Table IV, we have listed the percentage of the total number of atoms that has to be taken off a platelet (the size is irrelevant after normalization by the total number of atoms) to retain an SSA $=5000 \mathrm{~m}^{2} / \mathrm{g}$. Both 1-layer and 2-layer platelets are considered. Since the maximum number of atoms allowed to be stripped from a 1-layer platelet is more tolerable than that allowed from a 2-layer platelet, we think that a $5000-\mathrm{m}^{2} / \mathrm{g}$ ACF consists of relatively more 1-layer platelets, consistent with the fact that this carbon actually exists in powder form using a suitable carbon precursor.

The conductivity results are now discussed. If we assume that the dominant conduction mechanism at low temperature is hopping of some kind, then a high value of conductivity, being the consequence of a high probability of tunneling, will imply a large degree of disorder in the system. Based on this assumption, we observe in Fig. 2 that the amount of disorder increases with SSA and that the FRL fibers are more disordered than the FRS fibers. This agrees with our observations in the Raman scattering experiment described above. Further support can be found in the photoconductivity data obtained by Kuriyama and Dresselhaus. ${ }^{9}$ Their results show that both the photoconductivity and the decay time increase with SSA, with the decay time less dependent on SSA than the photoconductivity. This trend is internally consistent only if the photo-carrier mobility increases with SSA. Consequently, if the conduction by the photo-carriers is also governed by hopping at low

TABLE IV. Percentages of the total number of atoms (NM and $2 N M)$ taken away from platelets of 1 layer thick and also of 2 layers thick, respectively, as we increase the size ( $M$ number of atoms) of the pores within each layer. The maximum $M$ value corresponds to $N=1$. The SSA is taken to be $5000 \mathrm{~m}^{2} / \mathrm{g}$ and the size of the platelet is irrelevant for each model considered.

\begin{tabular}{lllll}
\hline \multicolumn{1}{c}{$M$} & 1 & 2 & 3 & Max. \\
\hline$N M(\%)$ for 1-layer platelets & 16 & 22 & 26 & 35 \\
$2 N M(\%)$ for 2-layer platelets & 23 & 30 & 34 & 46 \\
\hline
\end{tabular}

temperature, the disorder must increase with increasing SSA. The following discussion about the relationship between the conductivity data and disorder is based on these important findings.

As stated above, the strongly temperature-dependent conductivity $\sigma(T)$ follows Eq. (2) with $p=2$. This $p=2$ behavior can in general be explained by 1-D variable-range hopping conduction (VRHC), Coulombgap conduction (CGC), or charge-energy-limited tunneling conduction (CELTC).

In strongly localized systems, it may be energetically favorable for a charge carrier to hop to a localization site more distant than the nearest-neighboring site, especially at low temperature where there are few phonons of sufficient energy for a nearest-neighbor hop. If neighboring graphite platelets are actually touching in some preferred in-plane direction, thus forming a glassy-carbon ribbonlike structure, 1-D VRHC is possible.

In a very disordered solid, VRHC takes a different form as a result of the vanishing of the density of localized states at the Fermi level due to carrier-carrier interaction. ${ }^{28}$ In this situation, $p=2$ in Eq. (2) for both 2-D and 3-D systems. Such a quasi-gap is known as the Coulomb gap and is observed, for example, at very low temperature in heavily doped and compensated germanium with doping levels of $2 \times 10^{17} \mathrm{~cm}^{-3}$ or more. ${ }^{29}$

CELTC is commonly observed in granular metallic systems in which some charging energy is required for the generation of charge carriers. ${ }^{30}$ We are aware that there are newer models adapted to rid CELTC of its unfounded assumption that the grain size and the separation are correlated. As revealed by the TEM picture (Fig. 8), the microstructure in ACF's which consists of conducting basic structural units (BSU's) surrounded by dielectric media (micropores), is not too different from that of a granular metal.

In the following, each of these models is evaluated with respect to its physical parameters so that the correct model can be identified. Our approach is to make reasonable assumptions about the localization lengths in order to extract physical parameters such as the density of states near the Fermi level $[g(\mu)]$ or the dielectric constant, depending on the model used. For comparison, $T_{0}$ for only FRS15 ACF's is selected for more detailed calculations because they have a typical and intermediate room-temperature conductivity among all types of ACF's. In this process, CELTC is found to be the most reasonable conduction model.

We learn from our Raman scattering results that the in-plane size of a platelet is about $25 \AA$. A reasonable value for the localization length $\xi$ in each VRHC model should then be $\sim 12 \AA$, since the extent of the carrier wave function, which is generally assumed to be a symmetric hydrogenic ground-state wave function $F(r) \sim$ $\exp (-r / \xi)$, cannot exceed the size of the confining 
platelet. Indeed, although a smaller value for $\xi$ is equally plausible for a localization site due to a dangling bond or an impurity site on the boundary of a platelet, it will render the density of states at the Fermi level $g(\mu)$ unphysically large, as will be seen later. To extract the density of states, we therefore use $\xi=12 \AA$ and the following equation ${ }^{31}$ :

$$
T_{0} \simeq \frac{C}{\mathrm{k}_{\mathrm{B}} g(\mu) \xi^{d}}
$$

where $d$ is the dimensionality of the system, $g(\mu)$ the $d$-dimensional density of localized states at the Fermi level $(\mu)$, and $C=21.2,13.8,8$ for 3-D, 2-D, and 1-D VRHC, respectively. It is noteworthy that as the disorder increases with SSA, $\xi$ should decrease while $g(\mu)$ may increase. It is thus not immediately clear whether $T_{0}$ should increase or decrease with more disorder. This may account for the insensitivity of $T_{0}$ to SSA in our fits. However, in comparing the FRS to the FRL fibers, we note that $T_{0}$ for the FRL fibers is generally greater than that for the FRS fibers for all VRHC models.

Values for $g(\mu)$ obtained from the fits are listed in Table V. For completeness, the results for the models consisting of 2-D/3-D VRHC plus a thermal activation term are also listed. They all appear to be inconsistent with the ESR estimates ${ }^{32}$ that the unpaired spin concentration $(N)$ in the ACP30 fibers is $\sim 3.2 \times 10^{21} / \mathrm{g}$ and that in the ACP20 fibers is $\sim 1.8 \times 10^{20} / \mathrm{g}$. It is argued that the ESR values are only lower bounds to the concentration of localization sites since not only unpaired spins can act as localization centers and there could be localization sites not picked up by the ESR measurements. The simple conduction model $\sigma=n e \mu$, however, does yield a concentration of charge carriers

TABLE V. The chosen fitted values of $T_{0}$ (from data for the FRS15 fiber) and the corresponding extracted physical parameters including the dielectric constant $(\epsilon)$, the reciprocal decay length $(1 / \chi)$, and the effective barrier height $(\phi)$ for the charge-energy-limited tunneling conduction (CELTC) model, the dielectric constant for the Coulomb-gap conduction (CGC) model, and the $n$-dimensional density of states at the Fermi level $\left[g(\mu)\right.$ measured in $\left.\mathrm{cm}^{-n} \mathrm{eV}^{-1}\right]$ for the $n$-dimensional variable-range hopping conduction ( $n$ - $d$ VRHC) model with a thermal activation term. Also listed for each model is the optimum hopping distance $R_{m}$ at $4 \mathrm{~K}$.

\begin{tabular}{|c|c|c|c|}
\hline Model & $\begin{array}{c}T_{0} \\
(\mathrm{~K})\end{array}$ & $\begin{array}{c}\text { Extracted } \\
\text { parameters }\end{array}$ & $\begin{array}{c}R_{m}(\stackrel{4 \mathrm{~K}}{\mathrm{~K}}) \\
\quad(\AA)\end{array}$ \\
\hline CELTC & 360 & $\begin{array}{l}\epsilon \sim 6 \\
1 / \chi \sim 13.6 \AA \\
\phi \sim 0.25 \mathrm{eV}\end{array}$ & 32 \\
\hline CGC & 360 & $\epsilon \simeq 110$ & 71 \\
\hline 1-D VRHC & 360 & $g(\mu) \simeq 2.1 \times 10^{9} \mathrm{~cm}^{-1} \mathrm{eV}^{-1}$ & 23 \\
\hline 2-D VRHC & 8000 & $g(\mu) \simeq 1.4 \times 10^{15} \mathrm{~cm}^{-2} \mathrm{eV}^{-1}$ & 38 \\
\hline 3-D VRHC & 63900 & $g(\mu)=2.2 \times 10^{21} \mathrm{~cm}^{-3} \mathrm{eV}^{-1}$ & 54 \\
\hline
\end{tabular}

(n) of about $\sim 10^{21} \mathrm{~cm}^{-3}$ using the room-temperature conductivity data in Fig. 2 and assuming a carrier mobility of $1 \mathrm{~cm}^{2} / \mathrm{Vs}$ as found in a phenol resin, which has a conductivity and an activation temperature similar to those of the phenolic ACF's studied here. The photoconductivity study, ${ }^{9}$ which showed that the photocarriers excited $\sim 2 \mathrm{eV}$ above the Fermi level also exhibit hopping behavior, suggests that the energy width of the localization sites must be on the order of $1 \mathrm{eV}$. Dividing $n$ by $1 \mathrm{eV}$ gives $g(\mu)$ values consistent with those listed in Table V.

The large values for $g(\mu)$ extracted for the $n-D$ VRHC models are, however, in contradiction with the localization length $\xi$ assumed earlier. The $n-D g(\mu)$ $(n \leqslant 3)$ can be converted approximately to the average spacing between nearest-neighbor localization sites $\bar{R}$ by assuming an energy width for the localized states to be $\sim 1 \mathrm{eV}$ as follows:

$$
\bar{R} \simeq \frac{1}{\left(g_{n-D}(\mu) \times 1 \mathrm{eV}\right)^{1 / n}} \simeq \begin{cases}0.05 \AA & n=1 \\ 3 \AA & n=2 \\ 8 \AA & n=3\end{cases}
$$

Therefore, within the extent of a carrier wave function $(\xi \sim 12 \AA)$, many localization sites are strongly overlapping, thus eliminating the need for hopping. This inconsistency between $\bar{R}$ and $\xi$ cannot be resolved by choosing a larger value of $\xi$ because the ratio $\bar{R} / \xi<1$ is independent of $\xi$, as can be seen from Eqs. (8) and (9). We therefore conclude that the VRHC models cannot explain the conduction behavior in ACF's.

For CGC, the expression for $T_{0}$ is ${ }^{31}$ :

$$
T_{0}=\frac{2.8 e^{2}}{\mathrm{k}_{\mathrm{B}} \epsilon \xi}
$$

where $e$ is the charge of the carrier and $\epsilon$ the dielectric constant to be extracted from the data. As listed in Table $\mathrm{V}, \epsilon \simeq 110$ is too large to be physical.

For CELTC, a characteristic distance analogous to the localization length is $1 / \chi$, which is given by:

$$
\chi=\sqrt{\frac{2 m \phi}{\hbar^{2}}} .
$$

Here, $\chi$ is the reciprocal of the decay length of the carrier wave function, $\phi$ the effective barrier height, and $m$ the effective mass of the carrier, which is taken to be the valence-band effective mass of crystalline graphite (i.e., $m=0.084 m_{0}$, where $m_{0}$ is the free electron mass). The corresponding expression for $T_{0}$ is:

$$
T_{0}=\frac{4 \chi \alpha s E_{c}}{\mathrm{k}_{\mathrm{B}}}
$$

where $s$ is the platelet separation in the direction perpendicular to the tunneling cross section, and $E_{c}$ the energy 
required to generate a pair of fully dissociated, oppositely charged platelets. Because the effective tunneling distance can be shortened if neighboring platelets are tilted toward each other at one end, a factor $\alpha<1$ is included to take this into account. It is more common to use $C=\chi \alpha s E_{c}=\mathrm{k}_{\mathrm{B}} T_{0} / 4$ as the characteristic energy for granular metallic systems. For FRS15 fibers, $C \simeq 8 \mathrm{meV}$, which is low but still within a physically reasonable range when compared to values typical of granular metallic systems. ${ }^{30}$

In crystalline graphitic systems, charge carriers are more likely to travel in the transverse direction than in the out-of-plane direction as evidenced by the large ratio of the in-plane diffusive conductivity to the out-of-plane hopping conductivity. ${ }^{33}$ The presence of the micropores, which are mainly formed between neighboring graphite platelets in the $c$-direction, increases the interlayer separation and lowers the hopping probability in the $c$ direction further. On a local scale, it is possible that some neighboring platelets are approximately aligned in the in-plane direction, providing an easier path for hopping than across the layers in the out-of-plane direction. If we assume that the platelet is shaped like a circular disk of size $\pi(L / 2)^{2} \times t$ and if we further approximate the surrounding platelets in the in-plane direction by a concentric circular ring, we can then obtain an expression for $E_{c}$ which can be subsequently used to obtain an estimate for the dielectric constant $\epsilon$. Then, the charge energy $E_{c}$ is approximately given by:

$$
E_{c} \simeq 2 \times \frac{e^{2}}{\epsilon \beta L} \ln \left(1+\frac{2 s}{L}\right) \quad \text { (in cgs) }
$$

where

$$
\beta=t / L \simeq \frac{2 \times 3.35 \AA}{25 \AA} \simeq 0.27
$$

in which $t$ is assumed to be about two layers thick [suitable for 2000-m²/g ACF's; see Eq. (6)]. A multiplicative factor of 2 is needed in Eq. (13) for the generation of an electron-hole pair.

In the original CELTC model proposed for granular metallic systems by Sheng et al., ${ }^{30}$ the ratio of the granular separation $(s)$ to the granular size $(L)$ in any local region is assumed to be constant, thus rendering the quantity $s E_{c}$ a constant also. In our ACF system, the average in-plane separation between the platelets $\left(s_{0}\right)$ is assumed to be a single-atom vacancy (i.e., $s_{0} \simeq$ $1.4 \AA$ ) and $s / L \simeq 1.4 \AA / 25 \AA=0.056$. Using the experimental value of $T_{0}=360 \mathrm{~K}$, Eqs. (12) and (13) give $\epsilon /(\alpha \chi) \sim 81.7 \AA$, which can be satisfied by Eq. (13) by setting

$$
\begin{aligned}
\alpha & =1 \quad(s \text { cannot be smaller }), \\
\epsilon & =6, \\
1 / \chi & =13.6 \AA .
\end{aligned}
$$

Using this value of $\mathcal{X}$, we can obtain from Eq. (11) the effective barrier height $\phi \sim 0.25 \mathrm{eV}$, which is smaller than the work function of graphite $(\sim 4.4 \mathrm{eV})$, but the barrier is very likely to be lowered by the image force. As a check for the CELTC model, $\phi$ is always greater than $\mathrm{k}_{\mathrm{B}} T$ within the range of our measurement temperature. The $\epsilon$ value, although arbitrarily chosen, is selected to give a reasonable value for $1 / \chi$ without being unreasonably large. Compared with the 1-D VRHC and CGC models, the CELTC model gives more reasonable values for physical parameters and hence is more likely to be the correct model.

The optimum hopping distance $\left(R_{m}\right)$ is computed next. Expressions for $R_{m}$ for $d$-dimensional VRHC and CGC can be found in Refs. 31 and 34, and numerical values for $R_{m}$ are listed in Table $\mathrm{V}$ for completeness. It is not absolutely clear that the $R_{m}$ values are generally greater than twice the nearest-neighbor distance $(L)$, as required by VRHC.

For CELTC, an analogous hopping distance is the separation between platelets for the optimum hopping path $\left(s_{m}\right)$, given by ${ }^{30}$ :

$$
s_{m}=\frac{1}{4 \chi} \sqrt{T_{0} / T}
$$

where $\chi$ is defined in Eq. (11). We notice that $s_{m} \simeq$ $32 \AA$ at $4 \mathrm{~K}$ is quite large but $s_{m} \simeq 4 \AA$ at $300 \mathrm{~K}$. The decade-change in $s_{m}$ is a result of the CELTC model which assumes a wide distribution of particle sizes $L$. So far, there is no evidence supporting such a wide distribution of $L$ in ACF's, nor was there any evidence in Ref. 30 regarding the decade-wide distribution. It is also argued that there is no reason why $s / L$ should be constant. The large value of the calculated $s_{m}$ is therefore only an intrinsic property of the CELTC model and might not be connected with any physical reality.

We have shown that amongst all the models we studied, CELTC is the most likely. The $n-D$ VRHC models yield values for the physical parameters that are not within the physically admissible range. On the other hand, considering the crude approximations made in the CELTC model, we think that the extracted physical parameters are quite physical in terms of their orders of magnitude. Furthermore, the appearance of randomly oriented platelets in TEM micrographs supports this model more than the others.

A definite identification of the actual conduction model may be achieved by performing conductivity measurements in strong electric $(E)$ fields for these ACF's. For example, for a sufficiently strong $E$ field in a $d$-dimensional $(d \geqslant 2)$ VRHC system, the current $I$ is given by ${ }^{35}$ :

$$
\ln I \propto \frac{1}{E^{1 / d}} .
$$


The current-voltage relation for CELTC is also nonohmic though assuming a different form from Eq. (16), as given below ${ }^{30}$ :

$$
\sigma(E) \propto \exp \left[-\left(E_{0} / E\right)\right]
$$

where $E_{0}$ is some characteristic field. Hence, measurements of the I-V characteristics in strong $E$ fields should be very sensitive to the conduction mechanism.

\section{CONCLUSIONS}

Activated carbon fibers with SSA up to $3000 \mathrm{~m}^{2} / \mathrm{g}$ were studied by the Raman scattering technique and by low-temperature electrical conductivity measurements. While both experiments provide good characterization tools for these types of ACF's, the transport experiment proves to be more sensitive to SSA than the Raman experiment. However, the Raman study reveals the presence of a well-resolved graphitic peak, indicating that despite the disorder due to the small size of the platelets, the platelets themselves remain quite graphitic.

We found that disorder increases with increasing SSA and that the long phenolic ACF's are more disordered than the short phenolic ACF's and the pitch-based ACF's. Both the TEM and the Raman results show that a graphite platelet is approximately $20-30 \AA$ wide and 1-3 layers thick. The temperature dependence of the conductivity follows Mott's law with an exponent of $1 / 2$. The charge-energy-limited tunneling conduction model is considered to be the most likely conduction mechanism at low temperature since the variable-range hopping conduction models give nonphysical values for their physical parameters. A definite and correct identification of the low-temperature conduction mechanism, however, awaits the results of future tests such as electrical conductivity measurements in strong electric fields.

We have proposed a model to explain the high SSA of ACF's in both fiber and powder form. In particular, the worm-web model is important in that together with the platelet model, it forms the basis for all our transport models.

\section{ACKNOWLEDGMENTS}

We are grateful to Dr. D. E. Heiman of the National Magnet Lab., M.I.T., for generously allowing us to use his optical equipment. We thank Dr. S. L. di Vittorio of the Department of Materials Science and Engineering at M.I.T. for many enlightening discussions. We also acknowledge the Lawrence Livermore National Laboratory for support of this work under subcontract B130530.

\section{REFERENCES}

1. A. Nishino, Tanso (Carbon Society of Japan) 132, 57 (1988).

2. K. Kaneko and N. Shindo, Carbon 27, 815 (1989).

3. E. Tanaka, Fuel and Combustion 54, 241 (1987).

4. M. Smíšek and S. Černý, Active Carbon: Manufacture, Properties and Applications (American Elsevier Publishing Company, New York, 1967).

5. D. D. Saperstein, J. Phys. Chem. 90, 3883 (1986).

6. R. McIntosh, R. S. Haines, and G. C. Benson, J. Chem. Phys. 15, 17 (1947).

7. W. W. Smeltzer and R. McIntosh, Can. J. Chem. 31, 1239 (1953).

8. S. L. di Vittorio, M. S. Dresselhaus, M. Endo, J. P. Issi, L. Piraux, and V. Bayot, J, Mater. Res. 6, 778 (1991).

9. K. Kuriyama and M. S. Dresselhaus, J. Mater. Res. 6, 1040 (1991).

10. B.S. Elman, M. S. Dresselhaus, G. Dresselhaus, E. W. Maby, and H. Mazurek, Phys. Rev. B 24, 1027 (1981).

11. M.S. Dresselhaus and G. Dresselhaus, in Light Scattering in Solids III, Topics in Applied Physics, edited by M. Cardona and G. Güntherodt (Springer, Berlin, Heidelberg, 1982), Vol. 51, p. 3.

12. T. C. Chieu, M.S. Dresselhaus, and M. Endo, Phys. Rev. B 26, 5867 (1982).

13. J. R. Dacey, D. F. Quinn, and J. T. Gallagher, Carbon 4, 73 (1966).

14. F. Carmona, P. Delhaès, G. Keryer, and J.P. Manceau, Solid State Commun. 14, 1183 (1974)

15. T. Hanawa and J. Kakinoki, Carbon 1, 403 (1964).

16. J. Heremans, Carbon 23, 431 (1985).

17. P. Delhaès and F. Carmona, in Chemistry and Physics of Carbon, edited by P. L. Walker, Jr. and P. A. Thrower (Marcel Dekker, Inc., New York, 1981), Vol. 17, p. 89.

18. Dennis F. Baker and Robert H. Bragg, Phys. Rev. B 28, 2219 (1983).

19. N.F. Mott, Conduction in Non-Crystalline Materials (Oxford University Press, New York, 1987).

20. R. M. Hill, Phys. Status Solidi A35, K29 (1976).

21. A. G. Zabrodskii, Sov. Phys.-Semicond. 11, 345 (1977).

22. W. H. Press, B. P. Flannery, S. A. Teukolsky, and W. T. Vetterling, Numerical Recipes in C (Cambridge University Press, New York, 1988).

23. A. W. P. Fung, Characterization of Activated Carbon Fibers, Master's Thesis, Department of Electrical Engineering and Computer Science, MIT, 1991.

24. D. S. Knight and W. B. White, J. Mater. Res. 4, 385 (1989).

25. M. S. Dresselhaus, G. Dresselhaus, K. Sugihara, I. L. Spain, and H. A. Goldberg, Graphite Fibers and Filaments (Springer, Berlin, Heidelberg, 1988).

26. C. T. Chan, K. M. Ho, and W. A. Kamitak hara, Phys. Rev. B 36, 3499 (1987).

27. M. Endo (unpublished).

28. A. L. Efros and B. I. Shklovskii, J. Phys. C8, L49 (1975).

29. A. G. Zabrodskii, Sov. Phys.-Semicond. 14, 781 (1980).

30. B. Abeles, P. Sheng, M. D. Coutts, and Y. Arie, Adv. Phys. 24, 407 (1975).

31. B. I. Shklovskii and A. L. Efros, Electronic Properties of Doped Semiconductors (Springer, Berlin, Heidelberg, 1984).

32. S. L. di Vittorio, M.S. Dresselhaus, T. Enoki, M. Endo, and T. Nakajima, Phys. Rev. (1992), in preparation.

33. B. T. Kelly, Physics of Graphite (Applied Science, London, 1981).

34. C. J. Adkins, in Hopping and Related Phenomena, edited by $\mathbf{H}$. Fritzsche and M. Pollak (World Scientific Publishing Company, Singapore, 1990), p. 93.

35. B. I. Shklovskii, Sov. Phys.-Semicond. 6, 1964 (1973) 